Objectives: We undertook this study to explore the roles of DP1 in the development of OA and to evaluate the efficacy of a DP1 selective agonist in the treatment of $O A$.

Methods: We compared the development of aging-associated OA and destabilization of the medial meniscus (DMM)-induced OA in DP1-deficient (DP1-/-) and wild-type (WT) mice. The progression of OA was assessed by histology, immunohistochemistry, and microcomputed tomography (micro-CT). Cartilage explants from DP1-/- and WT mice were treated with interleukin-1 $\alpha$ (IL-1 $\alpha$ ) ex vivo, to evaluate proteoglycan degradation. The effect of intra-peritoneal administration of the DP1 selective agonist BW245C on OA progression was evaluated in WT mice.

Results: Compared to WT mice, DP1/- mice had exacerbated cartilage degradation in both models of $\mathrm{OA}$ and this was associated with increased expression of MMP-13, and ADAMTS-5. In addition, DP1-/- mice demonstrated enhanced subchondral bone changes. Cartilage explants from DP1-/- mice showed enhanced proteoglycan degradation following treatment with IL-1 $\alpha$. Intraperitoneal injection of BW245C attenuated the severity of DMM-induced cartilage degradation and bony changes in WT mice.

Conclusions: These findings indicate a critical role for DP1 signaling in OA pathogenesis. Modulation of DP1 functions may constitute a potential therapeutic target for the development of novel OA treatments.

Acknowledgements: This work was supported by the Canadian Institutes of Health Research (CIHR) Grant MOP-130293, the Arthritis Society, and the Fonds de la Recherche du Centre de Recherche du Centre Hospitalier de l'Université de Montréal (CRCHUM).

Disclosure of Interest: None declared

DOI: 10.1136/annrheumdis-2017-eular.6132

\section{OP0182 CONVERGENCE OF JOINT REPAIR AND PAIN PATHWAYS VIA NERVE GROWTH FACTOR AND P75 EXPRESSING MESENCHYMAL STEM CELLS- A NOVEL EXPLANATION FOR OSTEOARTHRITIS PROGRESSION WITH ANTI-NGF IN OSTEOARTHRITIS}

T. Baboolal ${ }^{1}$, S. Al Hinai ${ }^{1}$, E. Jones ${ }^{1}$, J. Reckless ${ }^{2}$, M. Foster ${ }^{3}$, R. Doyle ${ }^{4}$, K. af Forselles $^{3}$, S. Westbrook ${ }^{3}$, D. McGonagle ${ }^{1} .{ }^{1}$ Leeds Institute of Rheumatic and Musculoskeletal Medicine, University of Leeds, Leeds; ${ }^{2}$ Rxcelerate Ltd, Cambridge; ${ }^{3}$ Levicept Ltd; ${ }^{4}$ Tetrad Discovery Ltd, Ramsgate, United Kingdom

Background: Nerve growth factor (NGF) is a key regulator of pain and anti-NGF therapy reduces osteoarthritis $(\mathrm{OA})$ associated pain. However, anti-NGF therapy is associated with rapidly progressive OA (RPOA) [1]. In hip OA there is a 5 -fold increase in mesenchymal stem cells (MSCs) from MRI bone marrow (BM) lesions, areas associated with OA progression [2]. MSCs in such lesions are uniformly positive for the NGF receptor, p75, which is also linked to chemotaxis and proliferation in other stromal cell compartments [3].

Objectives: Evaluate anti-NGF treatment in monoiodoacetate (MIA) induced OA and test whether NGF influences human BM-MSC function.

Methods: Human tibial plateau (TP) bone was isolated from patients undergoing total knee replacement. OA was induced in male Wistar rats $(n=6)$ by intra-articular injection of $0.3 \mathrm{mg}$ MIA. Each animal was treated with subcutaneous injection of control human IgG or anti-NGF ( $3 \mathrm{mg} / \mathrm{kg}$ ) at Days 5, 10 and 15 . Human and animal tissues sections were prepared for histological analysis using H\&E staining and immunohistochemistry (IHC) using anti-p75 and anti-NGF antibodies. BM-MSCs were isolated from iliac crest aspirates and cultured under normal conditions. Expression of $\mathrm{p} 75$ was induced following overnight incubation with $400 \mathrm{mM}$ ethanol $(\mathrm{EtOH})$ and confirmed by flow cytometry. Proliferation was assessed $\pm \mathrm{EtOH}$ and $0-1 \mu \mathrm{g} / \mathrm{ml}$ NGF.

Results: Regions adjacent to cartilage loss in human TP showed abundant stromal proliferation, NGF and p75 immunoreactivity. In MIA model, by Day 18 there was substantial loss of cartilage, bone remodelling and stromal proliferation mimicking human disease. By Week 4 animals demonstrated unequal weight-

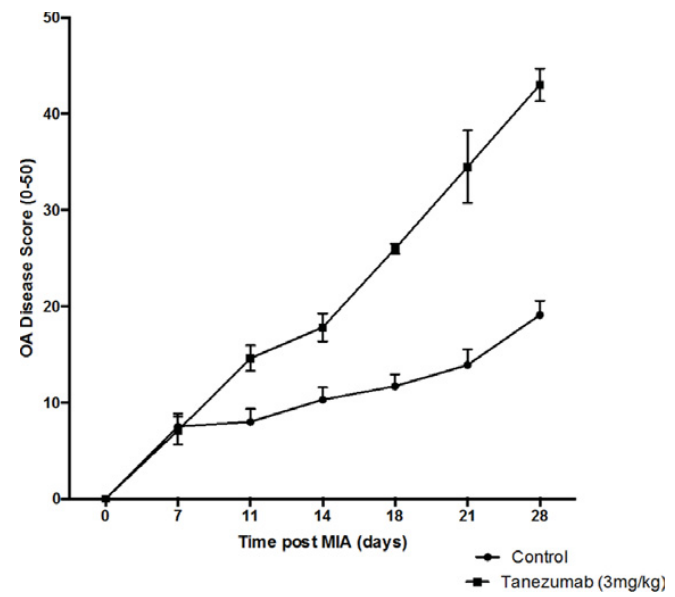

bearing $(p<0.05)$. Anti-NGF provided sufficient analgesia to normalise weightbearing. Compared to $\operatorname{lgG}$ control, arthropathy progression was faster (Fig) with complete cartilage loss, bone marrow necrosis and cyst formation by Day 21. p75 immunoreactivity was greatly reduced in MIA-injected rats receiving anti-NGF compared with IgG. NGF positive staining was widespread in naïve and MIA-injected knees, but almost absent from MIA-treated knees of anti-NGF treated rats.

To investigate increased $p 75$ positivity in knee OA, we restored p75 expression loss in expanded cell, to $97 \%$ of BM-MSCs $(n=3)$ using EtOH. Increased MSC proliferation was seen at Days 6 and $9(26 \%, p=0.03$ and $30 \%, p=0.01$ increase respectively, $n=7$ ) for $1 \mu \mathrm{g} / \mathrm{ml} \mathrm{NGF}$ in the presence of EtOH compared to control (no NGF). In cultures without EtOH induction (absent p75) NGF had no effect.

Conclusions: TP bone from OA patients and rat MIA-treated subchondral bone contains p75 positive staining in regions of cartilage destruction and associated NGF positivity. Anti-NGF treatment exacerbated MIA-induced OA and reduced NGF and p75 expression. In vitro, NGF increased BM-MSC proliferation, suggesting NGF may be involved in the stromal proliferation seen at sites of OA damage. Thus, NGF may regulate MSC function. Complete blockade represents a novel mechanism for accelerated joint destruction in OA.

References:

[1] Hochberg MC et al. Arthritis Rheumatol 2016.

[2] Campbell TM et al Arthritis Rheumatol 2016.

[3] Jiang Y, et al. Cartilag Arthritis Res Ther 2015

Disclosure of Interest: T. Baboolal: None declared, S. Al Hinai: None declared, E. Jones: None declared, J. Reckless Employee of: Rxcelerate Ltd, M. Foster Consultant for: Levicept Ltd, R. Doyle Employee of: Tetrad Discovery Ltd, K. af Forselles Consultant for: Levicept Ltd, S. Westbrook Shareholder of: Levicept Ltd, Employee of: Levicept Ltd, D. McGonagle: None declared

DOI: 10.1136/annrheumdis-2017-eular.6258

\section{OP0183 CORDYCEPIN, A NOVEL COMPOUND, REDUCES KNEE JOINT PATHOLOGY AND PAIN IN THE MONOSODIUM IODOACETATE (MIA) RAT MODEL OF OSTEOARTHRITIS}

S. Ashraf ${ }^{1}$, J. Burston ${ }^{2}$, V. Chapman ${ }^{2}$, C. de Moor ${ }^{1} .{ }^{1}$ Pharmacy; ${ }^{2}$ Arthritis Research UK Pain Centre, University of Nottingham, Nottingham, United Kingdom

Background: Cordycepin ( 3 ' deoxyadenosine) is a popular traditional medicine in Asia, taken for conditions associated with ageing. Osteoarthritis $(\mathrm{OA})$ is a common cause of pain and disability in the ageing population. Inflammation is a key component of osteoarthritic pain. Cordycepin is thought to act by inhibiting polyadenylation, the last step of mRNA synthesis, and can potentially have increased therapeutic benefit in OA (anti-inflammatory and analgesic) with fewer side effects than currently available therapies.

Objectives: The aim of this study was to determine whether cordycepin treatment alters osteoarthritic pain and pathology, and to decipher the mechanisms of action by which cordycepin exerts any potential beneficial actions.

Methods: OA was induced in male Sprague Dawley rats by intra-articular injection of mono-sodium iodoacetate (MIA; $1 \mathrm{mg} / 50 \mu \mathrm{l})$ on day 0 . Cordycepin was administered orally ( $2 \mathrm{mg} / \mathrm{rat}$ mixed in $1 \mathrm{~g}$ of wet mash) every other day for 2 weeks (pre-emptive study: day 0 to day 14, therapeutic study: day 14 to day 28). Pain behaviour was measured as hind-limb weight-bearing asymmetry and mechanical paw withdrawal thresholds. Joint tissues were collected at days 14 and 28. Joint changes were quantified using histology and immunohistochemistry techniques. Synovial inflammation was quantified as extent of CD68 positive macrophage and cellular infiltration. Synovial angiogenesis was measured as endothelial cells positive for proliferating cell nuclear antigen (PCNA). Safranin-O staining was used to score cartilage damage and bone changes (osteophytes and channels crossing the osteochondral junction [OCJ]). Tartrate-resistant acid phosphatase (TRAP) positive osteoclasts and ADAMTS- 5 and MMP13 positive chondrocytes were quantified as additional markers to detect bone and cartilage changes.

Results: The MIA rodent model of OA pain exhibited significant pain behaviour, synovial inflammation and angiogenesis, cartilage damage, osteophyte formation and subchondral bone changes, compared with non-arthritic controls. A two week pre-emptive and therapeutic treatment with cordycepin reduced MIA-induced pain behaviour and synovial changes (inflammation and angiogenesis). Pre-emptive cordycepin treatment reduced cartilage damage and the level of ADAMTS- 5 and MMP13 from the chondrocytes. Pre-emptive and therapeutic cordycepin treatment reduced the number of channels crossing the OCJ and TRAP positive osteoclasts in the subchondral bone, but had no effect on numbers of osteophytes. Therapeutic cordycepin treatment did not alter cartilage damage score or the level of ADAMTS- 5 and MMP13 positive chondrocytes.

Conclusions: Our data show that the analgesic effects of orally administered cordycepin in a pre-emptive and therapeutic protocol are associated with synovial changes (inflammation and angiogenesis) and bone remodelling. Administration of cordycepin before the onset of MIA-induced OA reduced cartilage damage and had a chondroprotective effect. Whereas therapeutically administered cordycepin did not alter cartilage damage. Further studies will investigate whether cordycepin mediated reduction in MIA-induced pathology and pain behaviour is as a result of its direct action on polyadenylation inhibition. Polyadenylation inhibitors could therefore be a novel class of drugs for treating OA.

Disclosure of Interest: None declared 